\title{
Lithium pollution of a white dwarf records the accretion of an extrasolar planetesimal
}

\author{
B. C. Kaiser ${ }^{1 *}$, J. C. Clemens' ${ }^{1}$, S. Blouin ${ }^{2}$, P. Dufour ${ }^{3,4}$, R. J. Hegedus ${ }^{1}$, J. S. Reding ${ }^{1}$, A. Bédard ${ }^{3}$ \\ ${ }^{1}$ Department of Physics and Astronomy, University of North Carolina, Chapel Hill, NC, USA. ${ }^{2}$ Los Alamos National Laboratory, Los Alamos, NM, USA. ${ }^{3}$ Département de \\ Physique, Université de Montréal, Montreal, QC, Canada. ${ }^{4}$ Institut de Recherche sur les Exoplanètes, Université de Montréal, Montreal, QC, Canada. \\ *Corresponding author. Email: ben.kaiser@unc.edu
}

Tidal disruption and subsequent accretion of planetesimals by white dwarfs can reveal the elemental abundances of rocky bodies in exoplanetary systems. Those abundances provide information on the composition of the nebula from which the systems formed, analogous to how meteorite abundances inform our understanding of the early Solar System. We report the detection of $\mathrm{Li}, \mathrm{Na}, \mathrm{K}$ and $\mathrm{Ca}$ in the atmosphere of the white dwarf Gaia DR2 4353607450860305024 , which we ascribe to accretion of a planetesimal. Using model atmospheres, we determine abundance ratios of these elements, and with the exception of $\mathrm{Li}$, they are consistent with meteoritic values in the Solar System. We compare the measured Li abundance to measurements in old stars and to expectations from Big Bang nucleosynthesis.

White dwarfs are remnants of main-sequence stars that have exhausted their available nuclear fuel and expelled their outer layers to leave a hot planet-sized object, which cools over billions of years. Their high surface gravities cause stratification of elements by mass, so undisturbed white dwarf atmospheres should exhibit spectral lines of only the lightest element present, usually hydrogen or helium. However, many white dwarf spectra show evidence for atmospheric contamination by heavier elements (referred to as pollution), in some cases accompanied by an excess in infrared emission due to a surrounding dust disk. These are attributed to the tidal disruption and accretion of extrasolar planetesimals (1-4).

Surveys indicate that up to half of hot white dwarfs show atmospheric pollution $(2,5,6)$ by elements that are expected to sink below the surface on timescales of $\sim$ days to $\sim$ Myrs (7), so planetesimal disruption and accretion must be a frequent event. In white dwarf atmospheres where the abundances of all major rock-forming elements have been measured, the extrasolar planetesimal compositions resemble those of the bulk Earth or other rocky Solar System bodies $[(8,9)$ compare (10)]. Abundances have mostly been measured from white dwarfs with effective temperatures $>4,500 \mathrm{~K}$, as cooler (therefore older) white dwarfs are faint and difficult to study $(9,11,12)$. A sample of 230 metal-polluted white dwarfs included only two with cooling ages $>7 \mathrm{Gyr}$, and most were younger than 4-5 Gyr (11). The Solar System is 4.5 Gyr old, so the compositions of exoplanets that formed at earlier times are unknown.

We observed the white dwarf Gaia DR2 4353607450860305024 (WD J164417.01-044947.7, hereafter WD J1644-0449) as part of a survey of ultra-cool objects selected from the Gaia Data Release 2 catalog $(13,14)$, chosen to have temperatures $<4,500 \mathrm{~K}$ and total (main-sequence + white dwarf cooling) ages $\gtrsim 7 \mathrm{Gyr}$. We expect elemental abundances of these systems to reflect Galactic chemical enrichment at their epoch of formation, as has been measured in the atmospheres of similarly old stars (15). WD J1644-0449 is not in previous white dwarf catalogs derived from Gaia data $(16,17)$ because its color is redder than the usual selection criteria. We obtained optical spectra of WD J1644-0449 using the Goodman Spectrograph mounted on the 4.1-m Southern Astrophysical Research (SOAR) telescope (18). We examined archival infrared photometry (18), finding no infrared excess indicative of a cool companion or dust disk.

Our spectra show (Fig. 1) that WD J1644-0449 is a white dwarf of spectral type DZ that exhibits several heavy element absorption features. The effective temperature is too low for the spectrum to show optical absorption lines of atomic $\mathrm{H}$ or He even if they dominate the atmosphere (as we expect). The dominant spectral feature is a broad and deep Na i D absorption line reminiscent of two previously known white dwarfs: WD J2356-209 and SDSS J133001.13+643523.7 (hereafter SDSS J1330+6435) $(19,20)$. Broad Ca ii $\mathrm{H}$ and $\mathrm{K}$ lines overlap a broad Ca i line, and a further broad dip is present at the wavelength of a molecular band of $\mathrm{MgH}$. We also identified a $\mathrm{K} \mathrm{i}$ line, and in two different instrument modes we detected an absorption feature centered at $6710 \AA$, which we identify as a $\mathrm{Li}$ i line with a rest wavelength of $6708 \AA$.

We examined published spectra of other white dwarfs known to have broad Na D lines $(19,20)$ and found that SDSS J1330+6435 also shows an absorption line at the location of the same Li line. This line was visible in a prior publication but not identified, perhaps due to the low signal-to-noise of 
the spectrum (21). WD J2356-209 does not show any evidence for Li absorption (19). We re-observed WD J2356-209 with the Goodman Spectrograph and again no Li or K lines were detected (fig. S3), but we placed upper limits on their abundances that are tighter than previously available (12).

To determine atmospheric abundances, we calculated a grid of white dwarf atmosphere models by adding Li to previously published models (22). We also employed models to evaluate the mass contained in the surface convection layer and the masses of accreted elements that are expected to be mixed in this convection zone. We used a theoretical massradius relation to estimate the stellar mass and radius consistently with all the data $(18,23)$. We estimated the temperature of WD J1644-0449 to be $3,830 \pm 230 \mathrm{~K}$, very cool for a metal-polluted white dwarf.

The detection of Li allows us to investigate the Li abundance of extrasolar planetesimals and to compare them to atmospheres of stars with similar age (24) and to the expectations of Li formation during Big Bang nucleosynthesis (BBN) (25). Li can be strongly depleted in stellar atmospheres, including in the Sun, because it is consumed by nuclear reactions at a lower temperature than $\mathrm{H}$. However, $\mathrm{Li}$ is incorporated into meteorites and planetesimals because it is only moderately volatile, condensing at higher temperatures than either $\mathrm{Na}$ or K. Thus measurements of Li polluted white dwarfs may offer a record of ancient Li abundances. However, the measurements must be corrected for a possible bias introduced by the different rates at which elements sink in a white dwarf atmosphere.

Planetesimal accretion onto a white dwarf occurs in three phases. In the increasing phase, the star is actively accreting material from one or more planetesimals, and the atmospheric abundance ratios equal those of the accreted body. If accretion continues for several elemental sinking timescales, an equilibrium between accretion and diffusion is reached in which atmospheric abundance ratios approach a steady-state value that differs from that of the accreted body, but which can be corrected using the ratio of the sinking timescales ( 7 , $10,18)$. Once all accretion stops, the atmospheric abundances decrease exponentially at rates that are generally slower for lighter elements; abundance ratios then depend both upon sinking times and the time elapsed since steady state accretion halted $(7,18)$. Figure 2 shows our measured abundance ratios, and corrected ratios for the steady-state and decreasing phases calculated using the sinking times for $\mathrm{Na}, \mathrm{K}$, and Ca. For reference we have also plotted abundance ratios for meteorites and a selection of Solar System bodies.

The K/Ca ratio in WD J1644-0449 remains nearly constant over accretion phases owing to the similar atomic masses and sinking times of these elements. Thus the accreted body had a $\mathrm{K} / \mathrm{Ca}$ ratio falling in a region centered between the carbonaceous Ivuna-type (CI) and carbonaceous
Mighei-type (CM) chondrite meteorites shown in Fig. 2, regardless of the accretion phase. Chondrites are the most primitive meteorites in the Solar System, and CI chondrites are used to establish the initial composition of the Solar nebula, based on their abundance similarities to many elements in the Solar atmosphere (26). Unlike K, Na sinks more slowly than $\mathrm{Ca}$ so the $\mathrm{Na} / \mathrm{Ca}$ ratio would be enhanced in the atmosphere during a decreasing phase of accretion. Figure 2 shows that the inferred $\mathrm{Na} / \mathrm{Ca}$ would in this case be lower than $\mathrm{CI}$ or $\mathrm{CM}$ chondrites and would deviate from the sequence defined by Solar System bodies. This sequence arises because K and $\mathrm{Na}$ have nearly identical condensation temperatures and are both lithophile elements (accumulate in the crust of differentiated bodies). Based on measurements of $\mathrm{Na} / \mathrm{Ca}$ in the atmospheres of old stars in the solar neighborhood, which show mean deviations of less than 0.2 dex from Solar ratios (27), we expect that the $\mathrm{Na} / \mathrm{Ca}$ abundance ratio in the gas from which WD J1644-0449 and its planetesimals formed is consistent with the Solar System value, within the uncertainties. Thus we expect the planetesimal abundances for $\mathrm{K} / \mathrm{Ca}$ and $\mathrm{Na} / \mathrm{Ca}$ fall along the same sequence as that defined by rocky bodies in the Solar System. This implies that the accretion is currently in a steady-state or early decreasing phase for WD J1644-0449. However, we also consider other accretion phases in our subsequent analysis.

The history of Li in the Galaxy is different from other elements and is more uncertain due to its destruction by nuclear burning in stars; in the solar atmosphere Li is depleted by two orders of magnitude compared to the CI chondrites (26). $\mathrm{BBN}$ theory predicts that a substantial amount of $\mathrm{Li}$ formed in the first 5 min after the Big Bang. The interstellar medium (ISM) abundance remained close to the BBN value until the ISM Fe content was enriched by explosions of massive stars to a value of $-1.0<[\mathrm{Fe} / \mathrm{H}]<-0.5$, where $[\mathrm{Fe} / \mathrm{H}]$ is the logarithm of the Fe to $\mathrm{H}$ ratio relative to the solar value (28). After that time, Li production by other nucleosynthetic processes increased the Li in the ISM (28). Ca was not produced during $\mathrm{BBN}$, but formed by subsequent stellar nucleosynthesis and injection into the ISM. Consequently, we expect the Li/Ca ratio to be highest in the very early Universe, because the $\mathrm{Ca}$ abundance was negligible while the Li abundance reflected the BBN value. Figure 3 shows Li/Ca measurements in atmospheres of typical stars in the solar neighborhood $(15,29)$ compared to the two Li-bearing white dwarfs. As expected, the highest $\mathrm{Li} / \mathrm{Ca}$ values measured are in the atmospheres of the oldest stars.

Figure 3 shows the inferred abundance ratios for the accreted bodies in WD J1644-0449 and SDSS J1330+6435 under the assumptions of steady-state and decreasing accretion phase (18). The steady state $\mathrm{Li} / \mathrm{Ca}$ is higher than stars of similar age, which could reflect Li depletion by nuclear reactions in the main-sequence stars. We also cannot rule out 
systematic differences in the age determination methods for white dwarfs and the nearby sample of stars. The latter are in some cases unphysically old; Fig. 3 includes several stars with inferred ages that are greater than the age of the Universe. Our white dwarf ages were calculated using published parameterizations of stellar lifetimes and white dwarf cooling ages, under the assumption of single-star evolution (18). The most likely mass of WD J1644-0449 is $0.45 \pm 0.12$ solar masses, which suggests that it may have lost mass through binary-star evolution, but there is no evidence of a companion. The $\mathrm{Li} / \mathrm{Ca}$ measurements are also higher than the CI chondrites, reflecting the lower abundance of $\mathrm{Ca}$ that prevailed at earlier times rather than an excess of Li over Solar values.

The lithium abundance history of the Galaxy is conventionally plotted on a Spite diagram (Fig. 4), which shows the $\mathrm{Li}$ abundance [defined as $\mathrm{A}(\mathrm{Li}) \equiv 12+\log (\mathrm{Li} / \mathrm{H})$ ] as a function of the iron abundance $[\mathrm{Fe} / \mathrm{H}]$, a proxy for age with lower values reflecting earlier epochs (30). Figure 4 shows a plateau at the expected $\mathrm{BBN}$ value of $\mathrm{A}(\mathrm{Li})$, followed by a rising segment that reflects later enrichment. The measured $\mathrm{A}(\mathrm{Li})$ in the old, low $[\mathrm{Fe} / \mathrm{H}]$ local stars under the plateau is lower than the $\mathrm{A}(\mathrm{Li})$ predicted by $\mathrm{BBN}$. The origin of this deficit is unknown, so it is commonly referred to as the cosmological Li problem (24).

As with Solar System meteorites (26), for the accreted planetesimals we cannot measure the $\mathrm{Li}$ to $\mathrm{H}$ ratio to get $\mathrm{A}(\mathrm{Li})$ directly. We employed published $\mathrm{Ca} / \mathrm{Fe}$ values measured from the atmospheres of the main Galactic stellar populations to convert our $\mathrm{Li} / \mathrm{Ca}$ into $\mathrm{Li} / \mathrm{Fe}$ values (18). Unlike $\mathrm{Li} / \mathrm{Ca}$ ratios, the $\mathrm{Ca} / \mathrm{Fe}$ values measured from main-sequence stellar atmospheres reflect the gas from which these stars formed, providing a sound basis for translating $\mathrm{Li} / \mathrm{Ca}$ to $\mathrm{Li} / \mathrm{Fe}$. Figure 4 shows $\mathrm{A}(\mathrm{Li})$ for each accreted extrasolar planetesimal as lines extending from $-1.5<[\mathrm{Fe} / \mathrm{H}]<0.24$. We have included representative lines for the inferred $\mathrm{A}(\mathrm{Li})$ for WD J1644-0449 and SDSS J1330+6435 for two possible Galactic stellar populations (18). Each population line has a different transformation for $\mathrm{Ca} / \mathrm{Fe}$, but both have an upward slope reflecting the increase in our calculated $\mathrm{A}(\mathrm{Li})$ that results from increasing $[\mathrm{Fe} / \mathrm{H}]$. We also illustrate the differing $\mathrm{A}(\mathrm{Li})$ inferred for steady-state accretion or decreasing phase accretion.

The accreted bodies in Fig. 4 extend at low metallicities to $\mathrm{A}(\mathrm{Li})$ values compatible with $\mathrm{BBN}$, but do not extend below that prediction. They do not show evidence for the cosmological $\mathrm{Li}$ problem exhibited by the local stars. Thus these Libearing extrasolar planetesimals represent an alternative to old stars for gaining insight into the primordial Li abundance, the earliest epochs of chemical enrichment in our Galaxy, and the properties of ancient exoplanets.

\section{REFERENCES AND NOTES}

1. M. Jura, A Tidally Disrupted Asteroid around the White Dwarf G29-38. Astrophys. J. 584, L91-L94 (2003). doi:10.1086/374036

2. B. Zuckerman, D. Koester, I. N. Reid, M. Hünsch, Metal Lines in DA White Dwarfs. Astrophys. J. 596, 477-495 (2003). doi:10.1086/377492

3. M. Jura, E. D. Young, Extrasolar Cosmochemistry. Annu. Rev. Earth Planet. Sci. 42, 45-67 (2014). doi:10.1146/annurev-earth-060313-054740

4. J. Farihi, Circumstellar debris and pollution at white dwarf stars. New Astron. Rev. 71, 9-34 (2016). doi:10.1016/i.newar.2016.03.001

5. B. Zuckerman, C. Melis, B. Klein, D. Koester, M. Jura, Ancient Planetary Systems are Orbiting a Large Fraction of White Dwarf Stars. Astrophys. J. 722, 725-736 (2010). doi:10.1088/0004-637X/722/1/725

6. D. Koester, B. T. Gänsicke, J. Farihi, The frequency of planetary debris around young white dwarfs. Astron. Astrophys. 566, A34 (2014). doi:10.1051/0004$6361 / 201423691$

7. D. Koester, Accretion and diffusion in white dwarfs: New diffusion timescales and applications to GD 362 and G 29-38. Astron. Astrophys. 498, 517-525 (2009). doi:10.1051/0004-6361/200811468

8. B. Klein, M. Jura, D. Koester, B. Zuckerman, C. Melis, Chemical Abundances in the Externally Polluted White Dwarf GD 40: Evidence of a Rocky Extrasolar Minor Planet. Astrophys. J. 709, 950-962 (2010). doi:10.1088/0004-637X/709/2/950

9. S. Xu, P. Dufour, B. Klein, C. Melis, N. N. Monson, B. Zuckerman, E. D. Young, M. A. Jura, Compositions of Planetary Debris around Dusty White Dwarfs. Astron. J. 158, 242 (2019). doi:10.3847/1538-3881/ab4cee

10. A. Swan, J. Farihi, D. Koester, M. Hollands, S. Parsons, P. W. Cauley, S. Redfield, B. T. Gänsicke, Interpretation and diversity of exoplanetary material orbiting white dwarfs. Mon. Not. R. Astron. Soc. 490, 202-218 (2019). doi:10.1093/mnras/stz2337

11. M. A. Hollands, D. Koester, V. Alekseev, E. L. Herbert, B. T. Gänsicke, Cool DZ white dwarfs - I. Identification and spectral analysis. Mon. Not. R. Astron. Soc. 467, 4970-5000 (2017). doi:10.1093/mnras/stx250

12. S. Blouin, P. Dufour, N. F. Allard, S. Salim, R. M. Rich, L. V. E. Koopmans, A New Generation of Cool White Dwarf Atmosphere Models. III. WD J2356-209: Accretion of a Planetesimal with an Unusual Composition. Astrophys. J. 872, 188 (2019). doi:10.3847/1538-4357/ab0081

13. Gaia Collaboration, T. Prusti, J. H. J. de Bruijne, A. G. A. Brown, A. Vallenari, C. Babusiaux, C. A. L. Bailer-Jones, U. Bastian, M. Biermann, D. W. Evans, L. Eyer, F. Jansen, C. Jordi, S. A. Klioner, U. Lammers, L. Lindegren, X. Luri, F. Mignard, D. J. Milligan, C. Panem, V. Poinsignon, D. Pourbaix, S. Randich, G. Sarri, P. Sartoretti, H. I. Siddiqui, C. Soubiran, V. Valette, F. van Leeuwen, N. A. Walton, C. Aerts, F. Arenou, M. Cropper, R. Drimmel, E. Høg, D. Katz, M. G. Lattanzi, W. O'Mullane, E. K. Grebel, A. D. Holland, C. Huc, X. Passot, L. Bramante, C. Cacciari, J. Castañeda, L. Chaoul, N. Cheek, F. De Angeli, C. Fabricius, R. Guerra, J. Hernández, A. JeanAntoine-Piccolo, E. Masana, R. Messineo, N. Mowlavi, K. Nienartowicz, D. Ordóñez-Blanco, P. Panuzzo, J. Portell, P. J. Richards, M. Riello, G. M. Seabroke, P. Tanga, F. Thévenin, J. Torra, S. G. Els, G. Gracia-Abril, G. Comoretto, M. GarciaReinaldos, T. Lock, E. Mercier, M. Altmann, R. Andrae, T. L. Astraatmadja, I. BellasVelidis, K. Benson, J. Berthier, R. Blomme, G. Busso, B. Carry, A. Cellino, G. Clementini, S. Cowell, O. Creevey, J. Cuypers, M. Davidson, J. De Ridder, A. de Torres, L. Delchambre, A. Dell'Oro, C. Ducourant, Y. Frémat, M. García-Torres, E. Gosset, J.-L. Halbwachs, N. C. Hambly, D. L. Harrison, M. Hauser, D. Hestroffer, S. T. Hodgkin, H. E. Huckle, A. Hutton, G. Jasniewicz, S. Jordan, M. Kontizas, A. J. Korn, A. C. Lanzafame, M. Manteiga, A. Moitinho, K. Muinonen, J. Osinde, E. Pancino, T. Pauwels, J.-M. Petit, A. Recio-Blanco, A. C. Robin, L. M. Sarro, C. Siopis, M. Smith, K. W. Smith, A. Sozzetti, W. Thuillot, W. van Reeven, Y. Viala, U. Abbas, A. Abreu Aramburu, S. Accart, J. J. Aguado, P. M. Allan, W. Allasia, G. Altavilla, M. A. Álvarez, J. Alves, R. I. Anderson, A. H. Andrei, E. Anglada Varela, E. Antiche, T. Antoja, S. Antón, B. Arcay, A. Atzei, L. Ayache, N. Bach, S. G. Baker, L. Balaguer-Núñez, C. Barache, C. Barata, A. Barbier, F. Barblan, M. Baroni, D. Barrado y Navascués, M. Barros, M. A. Barstow, U. Becciani, M. Bellazzini, G. Bellei, A. Bello García, V. Belokurov, P. Bendjoya, A. Berihuete, L. Bianchi, 0. Bienaymé, F. Billebaud, N. Blagorodnova, S. Blanco-Cuaresma, T. Boch, A. Bombrun, R. Borrachero, S. Bouquillon, G. Bourda, H. Bouy, A. Bragaglia, M. A. Breddels, N. Brouillet, T. Brüsemeister, B. Bucciarelli, F. Budnik, P. Burgess, R. Burgon, A. Burlacu, D. Busonero, R. Buzzi, E. Caffau, J. Cambras, H. Campbell, R. 
Cancelliere, T. Cantat-Gaudin, T. Carlucci, J. M. Carrasco, M. Castellani, P. Charlot, J. Charnas, P. Charvet, F. Chassat, A. Chiavassa, M. Clotet, G. Cocozza, R. S. Collins, P. Collins, G. Costigan, F. Crifo, N. J. G. Cross, M. Crosta, C. Crowley, C. Dafonte, Y. Damerdji, A. Dapergolas, P. David, M. David, P. De Cat, F. de Felice, P. de Laverny, F. De Luise, R. De March, D. de Martino, R. de Souza, J. Debosscher E. del Pozo, M. Delbo, A. Delgado, H. E. Delgado, F. di Marco, P. Di Matteo, S. Diakite, E. Distefano, C. Dolding, S. Dos Anjos, P. Drazinos, J. Durán, Y. Dzigan, E. Ecale, B. Edvardsson, H. Enke, M. Erdmann, D. Escolar, M. Espina, N. W. Evans, G. Eynard Bontemps, C. Fabre, M. Fabrizio, S. Faigler, A. J. Falcão, M. Farràs Casas, F. Faye, L. Federici, G. Fedorets, J. Fernández-Hernández, P. Fernique, A. Fienga, F. Figueras, F. Filippi, K. Findeisen, A. Fonti, M. Fouesneau, E. Fraile, M. Fraser, J. Fuchs, R. Furnell, M. Gai, S. Galleti, L. Galluccio, D. Garabato, F. García-Sedano, P. Garé, A. Garofalo, N. Garralda, P. Gavras, J. Gerssen, R. Geyer, G. Gilmore, S. Girona, G. Giuffrida, M. Gomes, A. González-Marcos, J. González-Núñez, J. J. González-Vidal, M. Granvik, A. Guerrier, P. Guillout, J. Guiraud, A. Gúrpide, R. Gutiérrez-Sánchez, L. P. Guy, R. Haigron, D. Hatzidimitriou, M. Haywood, U. Heiter, A. Helmi, D. Hobbs, W. Hofmann, B. Holl, G. Holland, J. A. S. Hunt, A. Hypki, V. Icardi, M. Irwin, G. Jevardat de Fombelle, P. Jofré, P. G. Jonker, A. Jorissen, F. Julbe, A. Karampelas, A. Kochoska, R. Kohley, K. Kolenberg, E. Kontizas, S. E. Koposov, G. Kordopatis, P. Koubsky, A. Kowalczyk, A. Krone-Martins, M. Kudryashova, I. Kull, R. K. Bachchan, F. Lacoste-Seris, A. F. Lanza, J.-B. Lavigne, C. Le Poncin-Lafitte, Y. Lebreton, T. Lebzelter, S. Leccia, N. Leclerc, I. LecoeurTaibi, V. Lemaitre, H. Lenhardt, F. Leroux, S. Liao, E. Licata, H. E. P. Lindstrøm, T. A. Lister, E. Livanou, A. Lobel, W. Löffler, M. López, A. Lopez-Lozano, D. Lorenz, T. Loureiro, I. MacDonald, T. Magalhães Fernandes, S. Managau, R. G. Mann, G. Mantelet, O. Marchal, J. M. Marchant, M. Marconi, J. Marie, S. Marinoni, P. M. Marrese, G. Marschalkó, D. J. Marshall, J. M. Martín-Fleitas, M. Martino, N. Mary, G. Matijevič, T. Mazeh, P. J. McMillan, S. Messina, A. Mestre, D. Michalik, N. R. Millar, B. M. H. Miranda, D. Molina, R. Molinaro, M. Molinaro, L. Molnár, M. Moniez, P. Montegriffo, D. Monteiro, R. Mor, A. Mora, R. Morbidelli, T. Morel, S. Morgenthaler, T. Morley, D. Morris, A. F. Mulone, T. Muraveva, I. Musella, J. Narbonne, G. Nelemans, L. Nicastro, L. Noval, C. Ordénovic, J. Ordieres-Meré, P. Osborne, C. Pagani, I. Pagano, F. Pailler, H. Palacin, L. Palaversa, P. Parsons, T. Paulsen, M. Pecoraro, R. Pedrosa, H. Pentikäinen, J. Pereira, B. Pichon, A. M. Piersimoni, F.-X. Pineau, E. Plachy, G. Plum, E. Poujoulet, A. Prša, L. Pulone, S. Ragaini, S. Rago, N. Rambaux, M. Ramos-Lerate, P. Ranalli, G. Rauw, A. Read, S. Regibo, F. Renk, C. Reylé, R. A. Ribeiro, L. Rimoldini, V. Ripepi, A. Riva, G. Rixon, M. Roelens, M. Romero-Gómez, N. Rowell, F. Royer, A. Rudolph, L. Ruiz-Dern, G. Sadowski, T. Sagristà Sellés, J. Sahlmann, J. Salgado, E. Salguero, M. Sarasso, H. Savietto, A. Schnorhk, M. Schultheis, E. Sciacca, M. Segol, J. C. Segovia, D. Segransan, E. Serpell, I.-C. Shih, R. Smareglia, R. L. Smart, C. Smith, E. Solano, F. Solitro, R. Sordo, S. Soria Nieto, J. Souchay, A. Spagna, F. Spoto, U. Stampa, I. A. Steele, H. Steidelmüller, C. A. Stephenson, H. Stoev, F. F. Suess, M. Süveges, J. Surdej, L. Szabados, E. Szegedi-Elek, D. Tapiador, F. Taris, G. Tauran, M. B. Taylor, R. Teixeira, D. Terrett, B. Tingley, S. C. Trager, C. Turon, A. Ulla, E. Utrilla, G. Valentini, A. van Elteren, E. Van Hemelryck, M. van Leeuwen, M. Varadi, A. Vecchiato, J. Veljanoski, T. Via, D. Vicente, S. Vogt, H. Voss, V. Votruba, S. Voutsinas, G. Walmsley, M. Weiler, K. Weingrill, D. Werner, T. Wevers, G. Whitehead, Ł. Wyrzykowski, A. Yoldas, M. Žerjal, S. Zucker, C. Zurbach, T. Zwitter, A. Alecu, M. Allen, C. Allende Prieto, A. Amorim, G. Anglada-Escudé, V. Arsenijevic, S. Azaz, P. Balm, M. Beck, H.-H. Bernstein, L. Bigot, A. Bijaoui, C. Blasco, M. Bonfigli, G. Bono, S. Boudreault, A. Bressan, S. Brown, P.-M. Brunet, P. Bunclark, R. Buonanno, A. G. Butkevich, C. Carret, C. Carrion, L. Chemin, F. Chéreau, L. Corcione, E. Darmigny, K. S. de Boer, P. de Teodoro, P. T. de Zeeuw, C. Delle Luche, C. D. Domingues, P. Dubath, F. Fodor, B. Frézouls, A. Fries, D. Fustes, D. Fyfe, E. Gallardo, J. Gallegos, D. Gardiol, M. Gebran, A. Gomboc, A. Gómez, E. Grux, A. Gueguen, A. Heyrovsky, J. Hoar, G. Iannicola, Y. Isasi Parache, A.-M. Janotto, E. Joliet, A. Jonckheere, R. Keil, D.-W. Kim, P. Klagyivik, J. Klar, J. Knude, 0. Kochukhov, I. Kolka, J. Kos, A. Kutka, V. Lainey, D. LeBouquin, C. Liu, D. Loreggia, V. V. Makarov, M. G. Marseille, C. Martayan, O. Martinez-Rubi, B. Massart, F. Meynadier, S. Mignot, U. Munari, A.-T. Nguyen, T. Nordlander, P. Ocvirk, K. S. O'Flaherty, A. Olias Sanz, P. Ortiz, J. Osorio, D. Oszkiewicz, A. Ouzounis, M. Palmer, P. Park, E. Pasquato, C. Peltzer, J. Peralta, F. Péturaud, T. Pieniluoma, E. Pigozzi, J. Poels, G. Prat, T. Prod'homme, F. Raison, J. M. Rebordao, D. Risquez, B. Rocca-Volmerange, S. Rosen, M. I. Ruiz-Fuertes, F. Russo, S. Sembay, I.
Serraller Vizcaino, A. Short, A. Siebert, H. Silva, D. Sinachopoulos, E. Slezak, M. Soffel, D. Sosnowska, V. Straižys, M. ter Linden, D. Terrell, S. Theil, C. Tiede, L. Troisi, P. Tsalmantza, D. Tur, M. Vaccari, F. Vachier, P. Valles, W. Van Hamme, L. Veltz, J. Virtanen, J.-M. Wallut, R. Wichmann, M. I. Wilkinson, H. Ziaeepour, S. Zschocke, The Gaia mission. Astron. Astrophys. 595, A1 (2016). doi:10.1051/0004-6361/201629272

14. L. Lindegren, J. Hernández, A. Bombrun, S. Klioner, U. Bastian, M. Ramos-Lerate, A. de Torres, H. Steidelmüller, C. Stephenson, D. Hobbs, U. Lammers, M. Biermann, R. Geyer, T. Hilger, D. Michalik, U. Stampa, P. J. McMillan, J. Castañeda, M. Clotet, G. Comoretto, M. Davidson, C. Fabricius, G. Gracia, N. C. Hambly, A. Hutton, A. Mora, J. Portell, F. van Leeuwen, U. Abbas, A. Abreu, M. Altmann, A. Andrei, E. Anglada, L. Balaguer-Núñez, C. Barache, U. Becciani, S. Bertone, L. Bianchi, S. Bouquillon, G. Bourda, T. Brüsemeister, B. Bucciarelli, D. Busonero, R. Buzzi, R. Cancelliere, T. Carlucci, P. Charlot, N. Cheek, M. Crosta, C. Crowley, J. de Bruijne, F. de Felice, R. Drimmel, P. Esquej, A. Fienga, E. Fraile, M. Gai, N. Garralda, J. J. González-Vidal, R. Guerra, M. Hauser, W. Hofmann, B. Holl, S. Jordan, M. G. Lattanzi, H. Lenhardt, S. Liao, E. Licata, T. Lister, W. Löffler, J. Marchant, J.-M. Martin-Fleitas, R. Messineo, F. Mignard, R. Morbidelli, E. Poggio, A. Riva, N. Rowell, E. Salguero, M. Sarasso, E. Sciacca, H. Siddiqui, R. L. Smart, A. Spagna, I. Steele, F. Taris, J. Torra, A. van Elteren, W. van Reeven, A. Vecchiato, Gaia Data Release 2. The astrometric solution. Astron. Astrophys. 616, A2 (2018). doi:10.1051/0004-6361/201832727

15. T. Bensby, S. Feltzing, M. S. Oey, Exploring the Milky Way stellar disk: A detailed elemental abundance study of $714 \mathrm{~F}$ and $\mathrm{G}$ dwarf stars in the solar neighbourhood. Astron. Astrophys. 562, A71 (2014). doi:10.1051/0004-6361/201322631

16. F. M. Jiménez-Esteban, S. Torres, A. Rebassa-Mansergas, G. Skorobogatov, E. Solano, C. Cantero, C. Rodrigo, A white dwarf catalogue from Gaia-DR2 and the Virtual Observatory. Mon. Not. R. Astron. Soc. 480, 4505-4518 (2018). doi:10.1093/mnras/sty2120

17. N. P. Gentile Fusillo, P.-E. Tremblay, B. T. Gänsicke, C. J. Manser, T. Cunningham, E. Cukanovaite, M. Hollands, T. Marsh, R. Raddi, S. Jordan, S. Toonen, S. Geier, M. Barstow, J. D. Cummings, A Gaia Data Release 2 catalogue of white dwarfs and a comparison with SDSS. Mon. Not. R. Astron. Soc. 482, 4570-4591 (2019). doi:10.1093/mnras/sty3016

18. Materials and methods are available as supplementary materials.

19. B. R. Oppenheimer, N. C. Hambly, A. P. Digby, S. T. Hodgkin, D. Saumon, Direct detection of galactic halo dark matter. Science 292, 698-702 (2001). doi:10.1126/science.1059954 Medline

20. H. C. Harris, J. Liebert, S. J. Kleinman, A. Nitta, S. F. Anderson, G. R. Knapp, J. Krzesiski, G. Schmidt, M. A. Strauss, D. Vanden Berk, D. Eisenstein, S. Hawley, B. Margon, J. A. Munn, N. M. Silvestri, J. A. Smith, P. Szkody, M. J. Collinge, C. C. Dahn, X. Fan, P. B. Hall, D. P. Schneider, J. Brinkmann, S. Burles, J. E. Gunn, G. S. Hennessy, R. Hindsley, Z. Ivezi, S. Kent, D. Q. Lamb, R. H. Lupton, R. C. Nichol, J. R. Pier, D. J. Schlegel, M. SubbaRao, A. Uomoto, B. Yanny, D. G. York, An Initial Survey of White Dwarfs in the Sloan Digital Sky Survey. Astron. J. 126, 1023-1040 (2003). doi:10.1086/376842

21. D. Homeier, N. Allard, F. Allard, P. H. Hauschildt, A. Schweitzer, P. C. Stencil, P. F. Weck, in 14th European Workshop on White Dwarfs, vol. 334 of Astronomical Society of the Pacific (ASP) Conference Series, D. Koester, S. Moehler, Eds. (ASP, 2005), pp. 209-214.

22. S. Blouin, P. Dufour, N. F. Allard, A New Generation of Cool White Dwarf Atmosphere Models. I. Theoretical Framework and Applications to DZ Stars. Astrophys. J. 863, 184 (2018). doi:10.3847/1538-4357/aad4a9

23. G. Fontaine, P. Brassard, P. Bergeron, The Potential of White Dwarf Cosmochronology. Publ. Astron. Soc. Pac. 113, 409-435 (2001). doi:10.1086/319535

24. M. Spite, F. Spite, Lithium abundance at the formation of the Galaxy. Nature 297. 483-485 (1982). doi:10.1038/297483a0

25. A. Coc, J.-P. Uzan, E. Vangioni, Standard big bang nucleosynthesis and primordial CNO abundances after Planck. J. Cosmol. Astropart. Phys. 2014, 050 (2014) doi:10.1088/1475-7516/2014/10/050

26. K. Lodders, H. Palme, H. P. Gail, in Astronomy, Astrophysics, and Cosmology: Solar System, vol. 4B, Landolt-Börnstein - Group VI Astronomy and Astrophysics, J. E. Trümper, Ed. (Springer, 2009), chap. 4.4.

27. N. R. Hinkel, F. X. Timmes, P. A. Young, M. D. Pagano, M. C. Turnbull, Stellar 
Abundances in the Solar Neighborhood: The Hypatia Catalog. Astron. J. 148, 54 (2014). doi:10.1088/0004-6256/148/3/54

28. V. Grisoni, F. Matteucci, D. Romano, X. Fu, Evolution of lithium in the Milky Way halo, discs, and bulge. Mon. Not. R. Astron. Soc. 489, 3539-3546 (2019). doi:10.1093/mnras/stz2428

29. T. Bensby, K. Lind, Exploring the production and depletion of lithium in the Milky Way stellar disk. Astron. Astrophys. 615, A151 (2018). doi:10.1051/0004$6361 / 201833118$

30. R. Rebolo, P. Molaro, J. E. Beckman, Lithium abundances in metal-deficient dwarfs. Astron. Astrophys. 192, 192-205 (1988).

31. S. Moehler, A. Modigliani, W. Freudling, N. Giammichele, A. Gianninas, A. Gonneau, W. Kausch, A. Lançon, S. Noll, T. Rauch, J. Vinther, Flux calibration of mediumresolution spectra from $300 \mathrm{~nm}$ to $2500 \mathrm{~nm}$ : Model reference spectra and telluric correction. Astron. Astrophys. 568, A9 (2014). doi:10.1051/0004$6361 / 201423790$

32. X. Fu, A. Bressan, P. Molaro, P. Marigo, Lithium evolution in metal-poor stars: From pre- main sequence to the Spite plateau. Mon. Not. R. Astron. Soc. 452, $3256-$ 3265 (2015). doi:10.1093/mnras/stv1384

33. A. Bouvier, M. Wadhwa, The age of the Solar System redefined by the oldest Pb$\mathrm{Pb}$ age of a meteoritic inclusion. Nat. Geosci. 3, 637-641 (2010). doi:10.1038/ngeo941

34. Planck Collaboration, P. A. R. Ade, N. Aghanim, M. Arnaud, M. Ashdown, J. Aumont, C. Baccigalupi, A. J. Banday, R. B. Barreiro, J. G. Bartlett, N. Bartolo, E. Battaner, R. Battye, K. Benabed, A. Benoît, A. Benoit-Lévy, J.-P. Bernard, M. Bersanelli, P. Bielewicz, J. J. Bock, A. Bonaldi, L. Bonavera, J. R. Bond, J. Borrill, F. R. Bouchet, F. Boulanger, M. Bucher, C. Burigana, R. C. Butler, E. Calabrese, J.-F. Cardoso, A. Catalano, A. Challinor, A. Chamballu, R.-R. Chary, H. C. Chiang, J. Chluba, P. R. Christensen, S. Church, D. L. Clements, S. Colombi, L. P. L. Colombo, C. Combet, A. Coulais, B. P. Crill, A. Curto, F. Cuttaia, L. Danese, R. D. Davies, R. J. Davis, P. de Bernardis, A. de Rosa, G. de Zotti, J. Delabrouille, F.-X. Désert, E. Di Valentino, C. Dickinson, J. M. Diego, K. Dolag, H. Dole, S. Donzelli, O. Doré, M. Douspis, A. Ducout, J. Dunkley, X. Dupac, G. Efstathiou, F. Elsner, T. A. Enßlin, H. K. Eriksen, M. Farhang, J. Fergusson, F. Finelli, O. Forni, M. Frailis, A. A. Fraisse, E. Franceschi, A. Frejsel, S. Galeotta, S. Galli, K. Ganga, C. Gauthier, M. Gerbino, T. Ghosh, M. Giard, Y. Giraud-Héraud, E. Giusarma, E. Gjerløw, J. González-Nuevo, K. M. Górski, S. Gratton, A. Gregorio, A. Gruppuso, J. E. Gudmundsson, J. Hamann, F. K. Hansen, D. Hanson, D. L. Harrison, G. Helou, S. Henrot-Versillé, C. HernándezMonteagudo, D. Herranz, S. R. Hildebrandt, E. Hivon, M. Hobson, W. A. Holmes, A. Hornstrup, W. Hovest, Z. Huang, K. M. Huffenberger, G. Hurier, A. H. Jaffe, T. R. Jaffe, W. C. Jones, M. Juvela, E. Keihänen, R. Keskitalo, T. S. Kisner, R. Kneissl, J. Knoche, L. Knox, M. Kunz, H. Kurki-Suonio, G. Lagache, A. Lähteenmäki, J.-M. Lamarre, A. Lasenby, M. Lattanzi, C. R. Lawrence, J. P. Leahy, R. Leonardi, J. Lesgourgues, F. Levrier, A. Lewis, M. Liguori, P. B. Lilje, M. Linden-Vørnle, M. López-Caniego, P. M. Lubin, J. F. Macías-Pérez, G. Maggio, D. Maino, N. Mandolesi, A. Mangilli, A. Marchini, M. Maris, P. G. Martin, M. Martinelli, E. Martínez-González, S. Masi, S. Matarrese, P. McGehee, P. R. Meinhold, A. Melchiorri, J.-B. Melin, L. Mendes, A. Mennella, M. Migliaccio, M. Millea, S. Mitra, M.-A. Miville-Deschênes, A. Moneti, L. Montier, G. Morgante, D. Mortlock, A. Moss, D. Munshi, J. A. Murphy, P. Naselsky, F. Nati, P. Natoli, C. B. Netterfield, H. U. Nørgaard-Nielsen, F. Noviello, D. Novikov, I. Novikov, C. A. Oxborrow, F. Paci, L. Pagano, F. Pajot, R. Paladini, D. Paoletti, B. Partridge, F. Pasian, G. Patanchon, T. J. Pearson, O. Perdereau, L. Perotto, F. Perrotta, V. Pettorino, F. Piacentini, M. Piat, E. Pierpaoli, D. Pietrobon, S. Plaszczynski, E. Pointecouteau, G. Polenta, L. Popa, G. W. Pratt, G. Prézeau, S. Prunet, J.-L. Puget, J. P. Rachen, W. T. Reach, R. Rebolo, M. Reinecke, M. Remazeilles, C. Renault, A. Renzi, I. Ristorcelli, G. Rocha, C. Rosset, M. Rossetti, G. Roudier, B. Rouillé d'Orfeuil, M. Rowan-Robinson, J. A. Rubiño-Martín, B. Rusholme, N. Said, V. Salvatelli, L. Salvati, M. Sandri, D. Santos, M. Savelainen, G. Savini, D. Scott, M. D. Seiffert, P. Serra, E. P. S. Shellard, L. D. Spencer, M. Spinelli, V. Stolyarov, R. Stompor, R. Sudiwala, R. Sunyaev, D. Sutton, A.-S. Suur-Uski, J.F. Sygnet, J. A. Tauber, L. Terenzi, L. Toffolatti, M. Tomasi, M. Tristram, T. Trombetti, M. Tucci, J. Tuovinen, M. Türler, G. Umana, L. Valenziano, J. Valiviita, F. Van Tent, P. Vielva, F. Villa, L. A. Wade, B. D. Wandelt, I. K. Wehus, M. White, S. D. M. White, A. Wilkinson, D. Yvon, A. Zacchei, A. Zonca, Planck 2015 results: XIII. Cosmological parameters. Astron. Astrophys. 594, A13 (2016). doi:10.1051/0004-6361/201525830
35. B. C. Kaiser, Lithium pollution of a white dwarf records the accretion of an extrasolar planetesimal (Reduction and Analysis Code), version 2, Zenodo (2020); http://doi.org/10.5281/zenodo.4244680.

36. S. Blouin, P. Dufour, A. Bédard, P. Bergeron, P. Brassard, G. Fontaine, Lithium pollution of a white dwarf records the accretion of an extrasolar planetesimal (Atmosphere/envelope code), version 1, Zenodo (2020); http://doi.org/10.5281/zenodo.4244665.

37. J. C. Clemens, J. A. Crain, R. Anderson, in Ground-Based Instrumentation for Astronomy, vol. 5492 of Society of Photo-Optical Instrumentation Engineers (SPIE) Conference Series, A. F. M. Moorwood, M. Iye, Eds. (SPIE, 2004), pp. 331340.

38. D. Tody, in Instrumentation in Astronomy VI, vol. 0627 of Society of Photo-Optical Instrumentation Engineers (SPIE) Conference Series, D. L. Crawford, Ed. (SPIE, 1986), pp. 733-748.

39. P. G. van Dokkum, Cosmic-Ray Rejection by Laplacian Edge Detection. Publ. Astron. Soc. Pac. 113, 1420-1427 (2001). doi:10.1086/323894

40. V. Kaufman, J. Sugar, Forbidden Lines in $n s^{2} n p^{k}$ Ground Configurations and $n s n p$ Excited Configurations of Beryllium through Molybdenum Atoms and lons. J. Phys. Chem. Ref. Data 15, 321-426 (1986). doi:10.1063/1.555775

41. P. Juncar, J. Pinard, J. Hamon, A. Chartier, Absolute Determination of the Wavelengths of the Sodium D1 and D2 Lines by Using a CW Tunable Dye Laser Stabilized on lodine. Metrologia 17, 77-79 (1981). doi:10.1088/0026$1394 / 17 / 3 / 001$

42. M. Stritzinger, N. B. Suntzeff, M. Hamuy, P. Challis, R. Demarco, L. Germany, A. M. Soderberg, An Atlas of Spectrophotometric Landolt Standard Stars. Publ. Astron. Soc. Pac. 117, 810-822 (2005). doi:10.1086/431468

43. D. Homeier, N. Allard, C. M. S. Johnas, P. H. Hauschildt, F. Allard, in 15th European Workshop on White Dwarfs, vol. 372 of Astronomical Society of the Pacific (ASP) Conference Series, R. Napiwotzki, M. R. Burleigh, Eds. (ASP, 2007), pp. 277-282.

44. A. Y. Kesseli, A. A. West, M. Veyette, B. Harrison, D. Feldman, J. J. Bochanski, An Empirical Template Library of Stellar Spectra for a Wide Range of Spectral Classes, Luminosity Classes, and Metallicities Using SDSS BOSS Spectra. Astrophys. J. 230, 16 (2017). doi:10.3847/1538-4365/aa656d

45. K. C. Chambers, E. A. Magnier, N. Metcalfe, H. A. Flewelling, M. E. Huber, C. Z. Waters, L. Denneau, P. W. Draper, D. Farrow, D. P. Finkbeiner, C. Holmberg, J. Koppenhoefer, P. A. Price, A. Rest, R. P. Saglia, E. F. Schlafly, S. J. Smartt, W. Sweeney, R. J. Wainscoat, W. S. Burgett, S. Chastel, T. Grav, J. N. Heasley, K. W. Hodapp, R. Jedicke, N. Kaiser, R.-P. Kudritzki, G. A. Luppino, R. H. Lupton, D. G. Monet, J. S. Morgan, P. M. Onaka, B. Shiao, C. W. Stubbs, J. L. Tonry, R. White, E. Bañados, E. F. Bell, R. Bender, E. J. Bernard, M. Boegner, F. Boffi, M. T. Botticella, A. Calamida, S. Casertano, W.-P. Chen, X. Chen, S. Cole, N. Deacon, C. Frenk, A. Fitzsimmons, S. Gezari, V. Gibbs, C. Goessl, T. Goggia, R. Gourgue, B. Goldman, P. Grant, E. K. Grebel, N. C. Hambly, G. Hasinger, A. F. Heavens, T. M. Heckman, R. Henderson, T. Henning, M. Holman, U. Hopp, W.-H. Ip, S. Isani, M. Jackson, C. D. Keyes, A. M. Koekemoer, R. Kotak, D. Le, D. Liska, K. S. Long, J. R. Lucey, M. Liu, N. F. Martin, G. Masci, B. McLean, E. Mindel, P. Misra, E. Morganson, D. N. A. Murphy, A. Obaika, G. Narayan, M. A. Nieto-Santisteban, P. Norberg, J. A. Peacock, E. A. Pier, M. Postman, N. Primak, C. Rae, A. Rai, A. Riess, A. Riffeser, H. W. Rix, S. Röser, R. Russel, L. Rutz, E. Schilbach, A. S. B. Schultz, D. Scolnic, L. Strolger, A. Szalay, S. Seitz, E. Small, K. W. Smith, D. R. Soderblom, P. Taylor, R. Thomson, A. N. Taylor, A. R. Thakar, J. Thiel, D. Thilker, D. Unger, Y. Urata, J. Valenti, J. Wagner, T. Walder, F. Walter, S. P. Watters, S. Werner, W. M. Wood-Vasey, R. Wyse, The Pan-STARRS1 Surveys. arXiv:1612.05560 [astro-ph.IM] (29 January 2019).

46. R. G. McMahon, M. Banerji, E. Gonzalez, S. E. Koposov, V. J. Bejar, N. Lodieu, R. Rebolo, VHS Collaboration, First Scientific Results from the VISTA Hemisphere Survey (VHS). Messenger 154, 35-37 (2013).

47. S. Blouin, P. Dufour, N. F. Allard, M. Kilic, A New Generation of Cool White Dwarf Atmosphere Models. II. A DZ Star with Collision-induced Absorption. Astrophys. J. 867, 161 (2018). doi:10.3847/1538-4357/aae53a

48. P. Dufour, P. Bergeron, J. Liebert, H. C. Harris, G. R. Knapp, S. F. Anderson, P. B. Hall, M. A. Strauss, M. J. Collinge, M. C. Edwards, On the Spectral Evolution of Cool, Helium-Atmosphere White Dwarfs: Detailed Spectroscopic and Photometric Analysis of DZ Stars. Astrophys. J. 663, 1291-1308 (2007). doi:10.1086/518468

49. S. Coutu, P. Dufour, P. Bergeron, S. Blouin, E. Loranger, N. F. Allard, B. H. Dunlap Analysis of Helium-rich White Dwarfs Polluted by Heavy Elements in the Gaia Era. 
Astrophys. J. 885, 74 (2019). doi:10.3847/1538-4357/ab46b9

50. S. Blouin, P. M. Kowalski, P. Dufour, Pressure Distortion of the $\mathrm{H}_{2}-\mathrm{He}$ Collisioninduced Absorption at the Photosphere of Cool White Dwarf Stars. Astrophys. J. 848, 36 (2017). doi:10.3847/1538-4357/aa8ad6

51. N. F. Allard, A. Royer, J. F. Kielkopf, N. Feautrier, Effect of the variation of electricdipole moments on the shape of pressure-broadened atomic spectral lines. Phys. Rev. A 60, 1021-1033 (1999). doi:10.1103/PhysRevA.60.1021

52. M. Tassoul, G. Fontaine, D. E. Winget, Evolutionary Models for Pulsation Studies of White Dwarfs. Astrophys. J. Suppl. Ser. 72, 335 (1990). doi:10.1086/191420

53. E. L. Wright, P. R. M. Eisenhardt, A. K. Mainzer, M. E. Ressler, R. M. Cutri, T. Jarrett, J. D. Kirkpatrick, D. Padgett, R. S. McMillan, M. Skrutskie, S. A. Stanford, M. Cohen, R. G. Walker, J. C. Mather, D. Leisawitz, T. N. Gautier, I. McLean, D. Benford, C. J. Lonsdale, A. Blain, B. Mendez, W. R. Irace, V. Duval, F. Liu, D. Royer, I. Heinrichsen, J. Howard, M. Shannon, M. Kendall, A. L. Walsh, M. Larsen, J. G. Cardon, S. Schick, M. Schwalm, M. Abid, B. Fabinsky, L. Naes, C.-W. Tsai, The Wide-field Infrared Survey Explorer (WISE): Mission Description and Initial On-orbit Performance. Astron. J. 140, 1868-1881 (2010). doi:10.1088/0004-6256/140/6/1868

54. A. Mainzer, J. Bauer, T. Grav, J. Masiero, R. M. Cutri, J. Dailey, P. Eisenhardt, R. S. McMillan, E. Wright, R. Walker, R. Jedicke, T. Spahr, D. Tholen, R. Alles, R. Beck, H. Brandenburg, T. Conrow, T. Evans, J. Fowler, T. Jarrett, K. Marsh, F. Masci, H. McCallon, S. Wheelock, M. Wittman, P. Wyatt, E. DeBaun, G. Elliott, D. Elsbury, T. Gautier, S. Gomillion, D. Leisawitz, C. Maleszewski, M. Micheli, A. Wilkins, Preliminary Results from NEOWISE: An Enhancement to the Wide-field Infrared Survey Explorer for Solar System Science. Astrophys. J. 731, 53 (2011). doi:10.1088/0004-637X/731/1/53

55. J. H. D. Harrison, A. Bonsor, N. Madhusudhan, Polluted white dwarfs: Constraints on the origin and geology of exoplanetary material. Mon. Not. R. Astron. Soc. 479, 3814-3841 (2018). doi:10.1093/mnras/sty1700

56. C. Paquette, C. Pelletier, G. Fontaine, G. Michaud, Diffusion in White Dwarfs: New Results and Comparative Study. Astrophys. J. Suppl. Ser. 61, 197 (1986). doi:10.1086/191112

57. G. Fontaine, P. Brassard, P. Dufour, P.-E. Tremblay, in 19th European Workshop on White Dwarfs, vol. 493 of Astronomical Society of the Pacific (ASP) Conference Series, P. Dufour, P. Bergeron, G. Fontaine, Eds. (ASP, 2015), pp. 113-116.

58. K. Kitts, K. Lodders, Survey and evaluation of eucrite bulk compositions. Meteorit. Planet. Sci. 33, A197-A213 (1998). doi:10.1111/i.1945-5100.1998.tb01334.x

59. F. Tera, O. Eugster, D. S. Burnett, G. J. Wasserburg, Comparative study of Li, $\mathrm{Na}$, $\mathrm{K}, \mathrm{Rb}, \mathrm{Cs}, \mathrm{Ca}$, Sr and Ba abundances in achondrites and in Apollo 11 lunar samples. Geochim. Cosmochim. Acta 1, 1637 (1970).

60. G. J. Taylor, The bulk composition of Mars. Geochem. 73, 401-420 (2013). doi:10.1016/i.chemer.2013.09.006

61. H. S. Wang, C. H. Lineweaver, T. R. Ireland, The elemental abundances (with uncertainties) of the most Earth-like planet. Icarus 299, 460-474 (2018). doi:10.1016/i.icarus.2017.08.024

62. J. R. Rumble, D. R. Lide, T. J. Bruno, CRC Handbook of Chemistry and Physics: A Ready-Reference Book of Chemical and Physical Data (CRC Press, ed. 100, 2019).

63. K. Lodders, B. Fegley, The Planetary Scientist's Companion (Oxford Univ. Press, 1998).

64. A. Bardyn, D. Baklouti, H. Cottin, N. Fray, C. Briois, J. Paquette, O. Stenzel, C. Engrand, H. Fischer, K. Hornung, R. Isnard, Y. Langevin, H. Lehto, L. Le Roy, N. Ligier, S. Merouane, P. Modica, F.-R. Orthous-Daunay, J. Rynö, R. Schulz, J. Silén, L. Thirkell, K. Varmuza, B. Zaprudin, J. Kissel, M. Hilchenbach, Carbon-rich dust in comet 67P/Churyumov-Gerasimenko measured by COSIMA/Rosetta. Mon. Not. R. Astron. Soc. 469, S712-S722 (2017). doi:10.1093/mnras/stx2640

65. J. D. Cummings, J. S. Kalirai, P. E. Tremblay, E. Ramirez-Ruiz, J. Choi, The White Dwarf Initial-Final Mass Relation for Progenitor Stars from 0.85 to $7.5 \mathrm{M}$. Astrophys. J. 866, 21 (2018). doi:10.3847/1538-4357/aadfd6

66. J. R. Hurley, O. R. Pols, C. A. Tout, Comprehensive analytic formulae for stellar evolution as a function of mass and metallicity. Mon. Not. R. Astron. Soc. 315 543-569 (2000). doi:10.1046/i.1365-8711.2000.03426.x

67. A. Gianninas, B. Curd, J. R. Thorstensen, M. Kilic, P. Bergeron, J. J. Andrews, P. Canton, M. A. Agüeros, Ultracool white dwarfs and the age of the Galactic disc. Mon. Not. R. Astron. Soc. 449, 3966-3980 (2015). doi:10.1093/mnras/stv545

68. M. Kilic, P. Bergeron, A. Kosakowski, W. R. Brown, M. A. Agüeros, S. Blouin, The 100 pc White Dwarf Sample in the SDSS Footprint. Astrophys. J. 898, 84 (2020). doi:10.3847/1538-4357/ab9b8d

69. S. Torres, C. Cantero, A. Rebassa-Mansergas, G. Skorobogatov, F. M. JiménezEsteban, E. Solano, Random Forest identification of the thin disc, thick disc, and halo Gaia-DR2 white dwarf population. Mon. Not. R. Astron. Soc. 485, 5573-5589 (2019). doi:10.1093/mnras/stz814

70. R. Schönrich, J. Binney, Origin and structure of the Galactic disc(s). Mon. Not. R. Astron. Soc. 399, 1145-1156 (2009). doi:10.1111/i.1365-2966.2009.15365.x

71. K. Hawkins, G. Kordopatis, G. Gilmore, T. Masseron, R. F. G. Wyse, G. Ruchti, 0. Bienaymé, J. Bland-Hawthorn, C. Boeche, K. Freeman, B. K. Gibson, E. K. Grebel, A. Helmi, A. Kunder, U. Munari, J. F. Navarro, Q. A. Parker, W. A. Reid, R. D. Scholz, G. Seabroke, A. Siebert, M. Steinmetz, F. Watson, T. Zwitter, Characterizing the high-velocity stars of RAVE: The discovery of a metal-rich halo star born in the Galactic disc. Mon. Not. R. Astron. Soc. 447, 2046-2058 (2015). doi:10.1093/mnras/stu2574

72. C. A. L. Bailer-Jones, J. Rybizki, M. Fouesneau, G. Mantelet, R. Andrae, Estimating Distance from Parallaxes. IV. Distances to 1.33 Billion Stars in Gaia Data Release 2. Astron. J. 156, 58 (2018). doi:10.3847/1538-3881/aacb21

73. V. V. Bobylev, Kinematics of the galaxy from Cepheids with proper motions from the Gaia DR1 catalogue. Astron. Lett. 43, 152-158 (2017). doi:10.1134/S106377371703001X

74. Astropy Collaboration, A. M. Price-Whelan, B. M. Sipőcz, H. M. Günther, P. L. Lim, S. M. Crawford, S. Conseil, D. L. Shupe, M. W. Craig, N. Dencheva, A. Ginsburg, J. T. VanderPlas, L. D. Bradley, D. Pérez-Suárez, M. de Val-Borro, T. L. Aldcroft, K. L. Cruz, T. P. Robitaille, E. J. Tollerud, C. Ardelean, T. Babej, Y. P. Bach, M. Bachetti, A. V. Bakanov, S. P. Bamford, G. Barentsen, P. Barmby, A. Baumbach, K. L. Berry, F. Biscani, M. Boquien, K. A. Bostroem, L. G. Bouma, G. B. Brammer, E. M. Bray, H. Breytenbach, H. Buddelmeijer, D. J. Burke, G. Calderone, J. L. C. Rodríguez, M. Cara, J. V. M. Cardoso, S. Cheedella, Y. Copin, L. Corrales, D. Crichton, D. D’Avella, C. Deil, É. Depagne, J. P. Dietrich, A. Donath, M. Droettboom, N. Earl, T. Erben, S. Fabbro, L. A. Ferreira, T. Finethy, R. T. Fox, L. H. Garrison, S. L. J. Gibbons, D. A. Goldstein, R. Gommers, J. P. Greco, P. Greenfield, A. M. Groener, F. Grollier, A. Hagen, P. Hirst, D. Homeier, A. J. Horton, G. Hosseinzadeh, L. Hu, J. S. Hunkeler, Ž. Ivezić, A. Jain, T. Jenness, G. Kanarek, S. Kendrew, N. S. Kern, W. E. Kerzendorf, A. Khvalko, J. King, D. Kirkby, A. M. Kulkarni, A. Kumar, A. Lee, D. Lenz, S. P. Littlefair, Z. Ma, D. M. Macleod, M. Mastropietro, C. McCully, S. Montagnac, B. M. Morris, M. Mueller, S. J. Mumford, D. Muna, N. A. Murphy, S. Nelson, G. H. Nguyen, J. P. Ninan, M. Nöthe, S. Ogaz, S. Oh, J. K. Parejko, N. Parley, S. Pascual, R. Patil, A. A. Patil, A. L. Plunkett, J. X. Prochaska, T. Rastogi, V. R. Janga, J. Sabater, P. Sakurikar, M. Seifert, L. E. Sherbert, H. Sherwood-Taylor, A. Y. Shih, J. Sick, M. T. Silbiger, S. Singanamalla, L. P. Singer, P. H. Sladen, K. A. Sooley, S. Sornarajah, O. Streicher, P. Teuben, S. W. Thomas, G. R. Tremblay, J. E. H. Turner, V. Terrón, M. H. Kerkwijk, A. de la Vega, L. L. Watkins, B. A. Weaver, J. B. Whitmore, J. Woillez, V. Zabalza, The Astropy Project: Building an Open-science Project and Status of the v2.0 Core Package. Astron. J. 156, 123 (2018). doi:10.3847/15383881/aabc4f

75. L. I. Mashonkina, M. D. Neretina, T. M. Sitnova, Y. V. Pakhomov, Abundances of $\alpha$ Process Elements in Thin-Disk, Thick-Disk, and Halo Stars of the Galaxy: Non-LTE Analysis. Astron. Rep. 63, 726-738 (2019). doi:10.1134/S1063772919090063

76. A. Bonsor, P. J. Carter, M. Hollands, B. T. Gänsicke, Z. Leinhardt, J. H. D. Harrison, Are exoplanetesimals differentiated? Mon. Not. R. Astron. Soc. 492, 2683-2697 (2020). doi:10.1093/mnras/stz3603

77. S. Blouin, N. F. Allard, T. Leininger, F. X. Gadéa, P. Dufour, Line Profiles of the Calcium I Resonance Line in Cool Metal-polluted White Dwarfs. Astrophys. J. 875 137 (2019). doi:10.3847/1538-4357/ab1266

\section{ACKNOWLEDGMENTS}

We benefited from conversations with M. Wadhwa and A. Mann. Based on observations obtained at the Southern Astrophysical Research (SOAR) telescope, which is a joint project of the Ministério da Ciência, Tecnologia, Inovações e Comunicações do Brasil (MCTIC/LNA), the US National Science Foundation's National Optical-Infrared Astronomy Research Laboratory (NOIRLab), the University of North Carolina at Chapel Hill (UNC), and Michigan State University (MSU). This work has made use of data from the European Space Agency (ESA) mission Gaia (https://www.cosmos.esa.int/gaia), processed by the Gaia Data Processing and Analysis Consortium (DPAC, 
https://www.cosmos.esa.int/web/gaia/dpac/consortium). Funding for the DPAC has been provided by national institutions, in particular the institutions participating in the Gaia Multilateral Agreement. The Pan-STARRS1 Surveys (PS1) and the PS1 public science archive have been made possible through contributions by the Institute for Astronomy, the University of Hawaii, the PanSTARRS Project Office, the Max-Planck Society and its participating institutes, the Max Planck Institute for Astronomy, Heidelberg and the Max Planck Institute for Extraterrestrial Physics, Garching, The Johns Hopkins University, Durham University, the University of Edinburgh, the Queen's University Belfast, the Harvard-Smithsonian Center for Astrophysics, the Las Cumbres Observatory Global Telescope Network Incorporated, the National Central University of Taiwan, the Space Telescope Science Institute, the National Aeronautics and Space Administration under Grant No. NNX08AR22G issued through the Planetary Science Division of the NASA Science Mission Directorate, the National Science Foundation Grant No. AST-1238877, the University of Maryland, Eotvos Lorand University (ELTE), the Los Alamos National Laboratory, and the Gordon and Betty Moore Foundation. Funding for the Sloan Digital Sky Survey (SDSS) has been provided by the Alfred P. Sloan Foundation, the Participating Institutions, the National Aeronautics and Space Administration, the National Science Foundation, the U.S. Department of Energy, the Japanese Monbukagakusho, and the Max Planck Society. The SDSS is a joint project of the University of Chicago, Fermilab, the Institute for Advanced Study, the Japan Participation Group, the Johns Hopkins University, Los Alamos National Laboratory, the Max Planck Institute for Astronomy (MPIA), the Max Planck Institute for Astrophysics (MPA), New Mexico State University, the University of Pittsburgh, Princeton University, the US Naval Observatory, and the University of Washington. Funding: S.B. acknowledges support from the Laboratory Directed Research and Development program of Los Alamos National Laboratory under project number 20190624PRD2. A.B. acknowledges support from NSERC (Canada) and the FRQNT (Québec). Author contributions: B.C.K. co-wrote the manuscript, obtained observations, co-identified the lithium line, and performed all calculations except the atmospheric and envelope modeling. J.C.C. co-wrote the manuscript, co-identified the lithium line, and supervised all calculations except the atmospheric and envelope modeling. S.B. performed the atmospheric modeling calculations, and co-wrote the manuscript. P.D. assisted with the atmospheric modeling calculations. R.J.H. obtained observations and assisted with the lithium line identification. J.S.R. obtained observations. A.B. performed the envelope modeling co-wrote the supplement. Competing interests: We declare no competing interests. Data and materials availability: The reduced Goodman spectra of WD J1644-0449 and WD J2356-209 are available in data S1 to S5. The SDSS J1330+6435 spectra are available from the Sloan Digital Sky Survey archive (https://dr9.sdss.org/basicSpectra) with plate 0603, modified Julian date 52056 and fiber 0510 . Our data reduction and analysis code is available (35). The atmosphere and envelope modeling software was written by a combination of authors (S.B. and P. D.) and non-authors (Gilles Fontaine, Pierre Brassard and Pierre Bergeron), so we cannot distribute the source code. An executable version with adjustable input parameters is available (36).

\section{SUPPLEMENTARY MATERIALS}

science.sciencemag.org/cgi/content/full/science.abd1714/DC1

Materials and Methods

Supplementary Text

Figs. S1 to S7

Tables S1 to S5

References (37-77)

Data S1 to S5

30 June 2020; accepted 4 December 2020

Published online 17 December 2020

10.1126/science.abd1714 


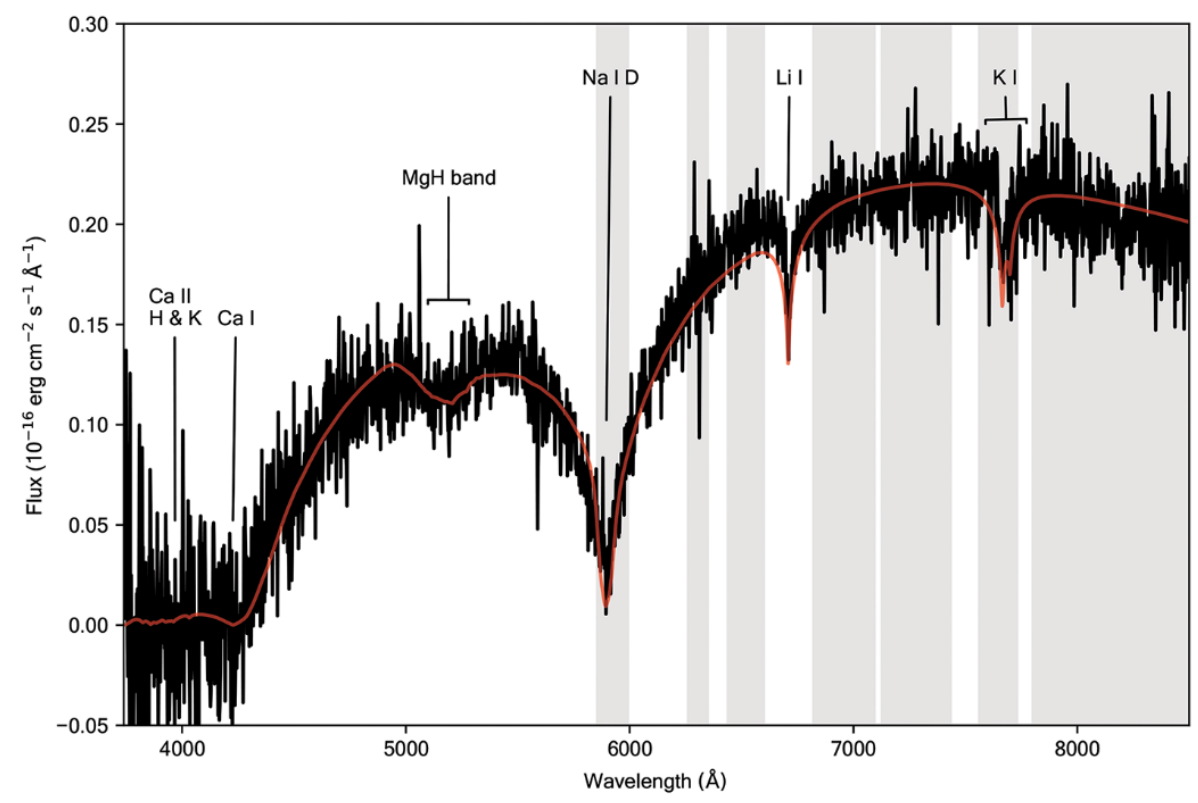

Fig. 1. Spectrum of WD J1644-0449. Data (black) are overlain with the best fitting model (red). The spectrum was constructed by combining two observations above and below $6800 \AA$ (18). Grey bands show regions of telluric absorption from the Earth's atmosphere (31); we applied telluric corrections at wavelengths $>6800 \AA$. Labeled absorption lines include $\mathrm{Ca}$ ii $\mathrm{H}$ and $\mathrm{K}(3934 \AA$ and $3969 \AA$ ), Ca i (4226 $\AA$ ), MgH band

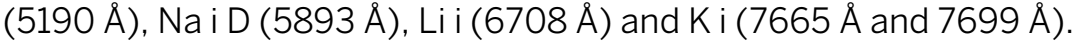




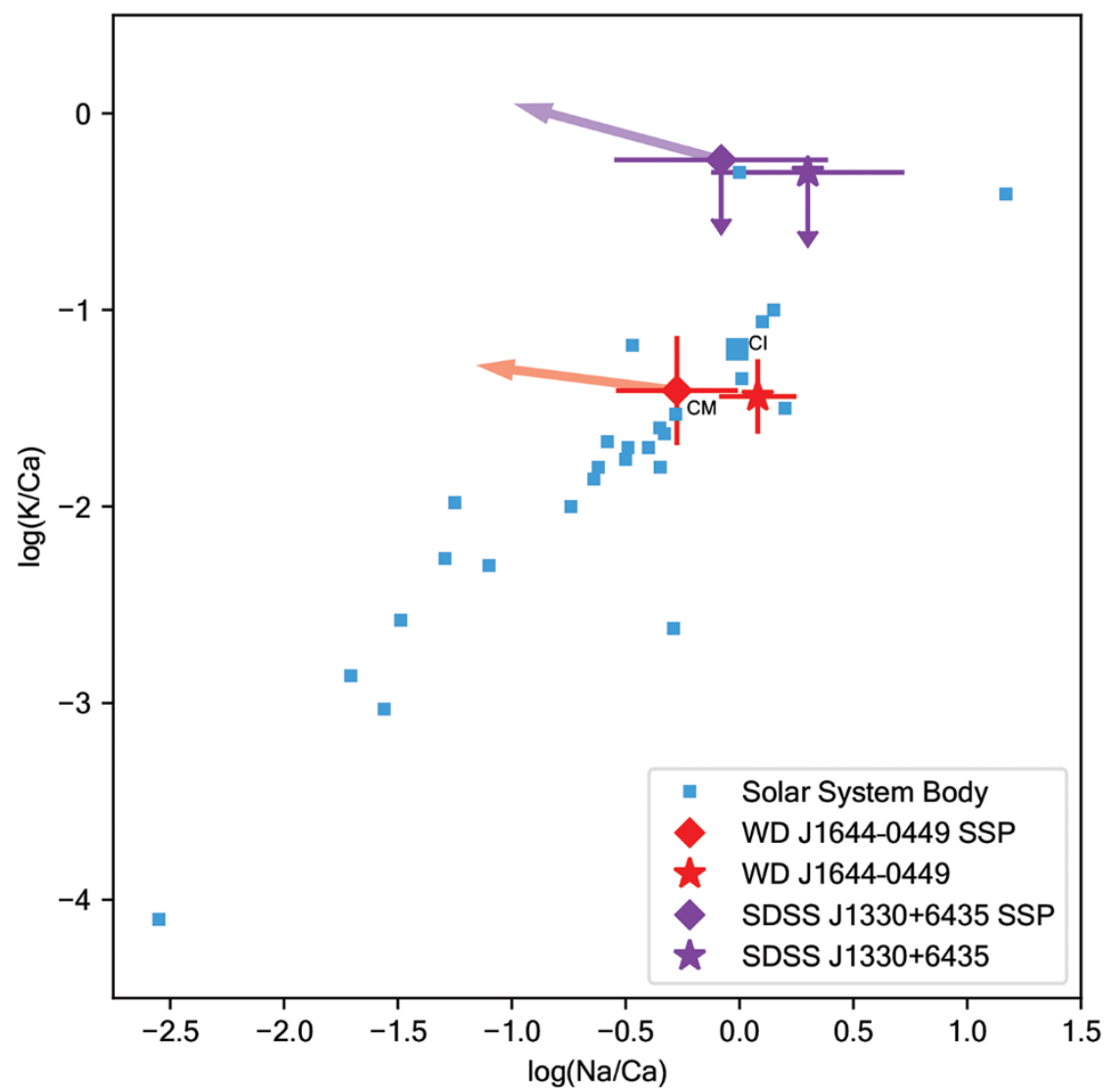

Fig. 2. Abundance ratios $\mathrm{K} / \mathrm{Ca}$ and $\mathrm{Na} / \mathrm{Ca}$ in the white dwarf planetesimals and Solar System bodies. Logarithmic number abundances for the white dwarfs WD J1644-0449 (red) and SDSS J1330+6435 (purple) are compared to Solar System bodies (blue), including meteorites (18). The two chondrite groups discussed in the text are labeled. Stars show the measured atmospheric abundances and diamonds show the inferred accreted body abundances, assuming steady-state accretion phase (SSP). Downward arrows indicate upper limits. Leftward arrows show corrections to the inferred abundance ratios if the accretion has been in the decreasing phase for 5 Ca sinking times (see table S4) in the style of prior work (10). Error bars show 1- $\sigma$ uncertainties (18). 


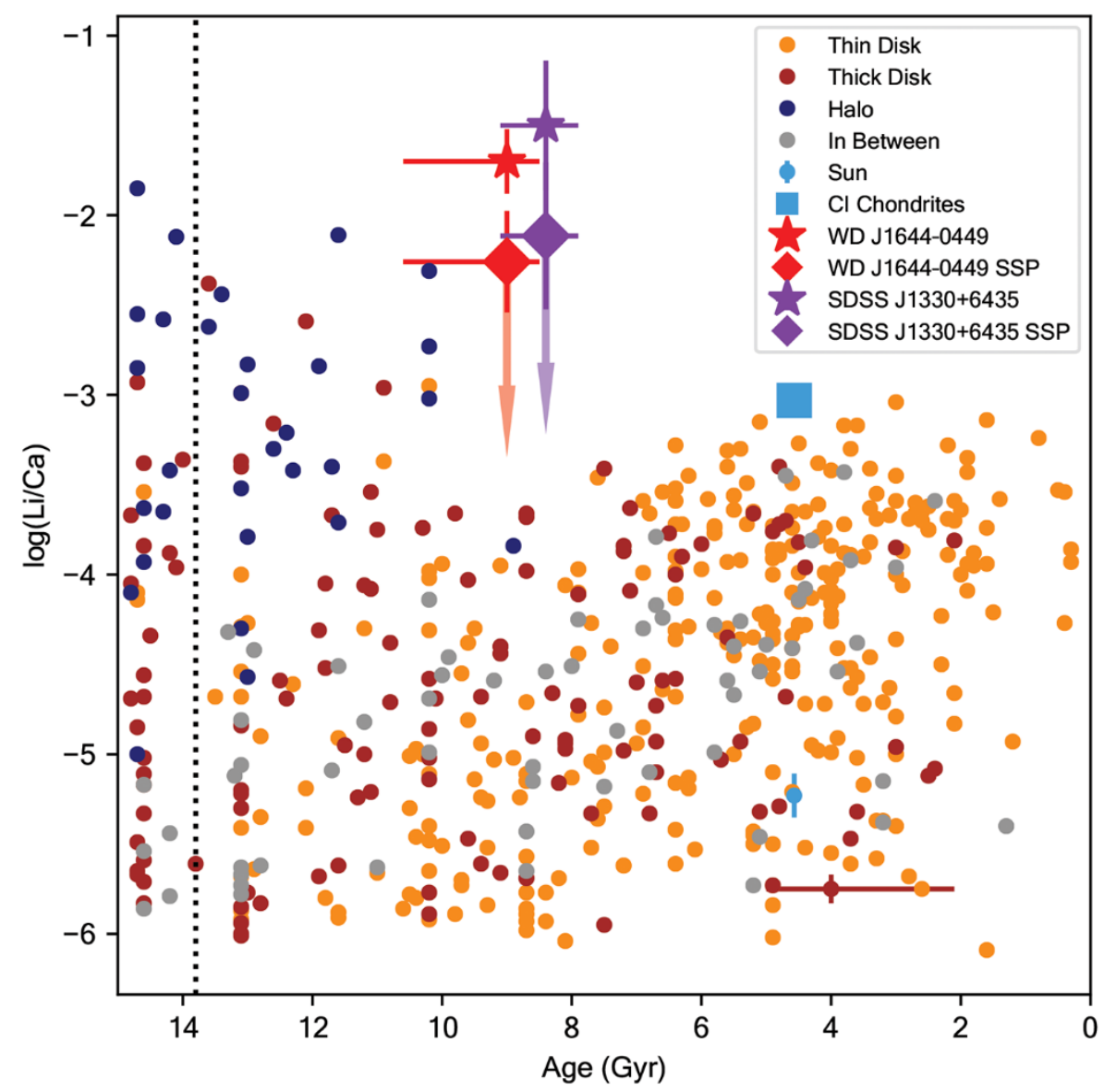

Fig. 3. Li/Ca evolution in the solar neighborhood. Logarithmic $\mathrm{Li} / \mathrm{Ca}$ is shown as a function of age for a sample of typical stars from the Solar neighborhood (circles), error bars in the lower right show typical 1- $\sigma$ uncertainties $(15,29)$. Because $\mathrm{Li}$ is consumed in stars, the highest values of $\log (\mathrm{Li} / \mathrm{Ca})$ at each age represent the best proxy for interstellar gas values (32). The atmospheric values for the Li-polluted white dwarfs are shown with the same symbols as Fig. 2 (18). White dwarf vertical error bars correspond to $1-\sigma$ uncertainty; horizontal error bars correspond to the $68 \%$ confidence interval. $\mathrm{Cl}$ chondrites (blue square, $1-\sigma$ vertical error bars are smaller than the symbol) represent the initial value for the Solar System, which is greater than the Sun's atmosphere (blue circle with vertical 1- $\sigma$ error bars) $(26,33)$. The age of the Universe is marked with the vertical dotted line (34). 


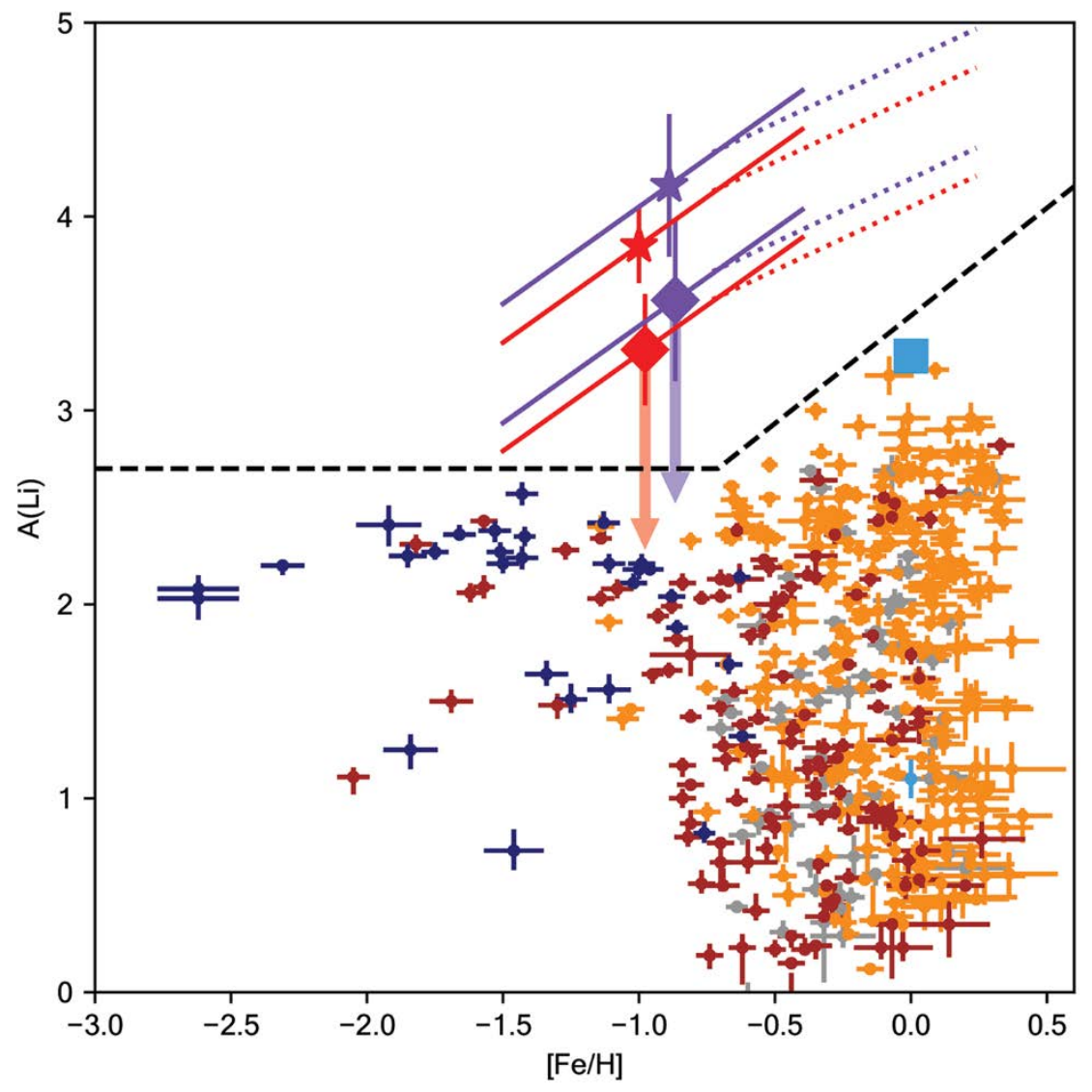

Fig. 4. Spite diagram for the same sources as shown in previous figure. Lithium abundance $A(L i)$ is shown as a function of iron abundance $[\mathrm{Fe} / \mathrm{H}]$. Predicted values for BBN and expected Galactic Li enrichment history are shown by the dashed line $(25,28)$. Symbols are the same as in Fig. 3. Sloped lines for each white dwarf represent abundances rescaled to $\mathrm{A}(\mathrm{Li})$ using $\log (\mathrm{Ca} / \mathrm{Fe})$ relations for thick disk (solid lines) and thin disk (dotted lines) Galactic stellar populations, extending over the full range of those populations (18). The white dwarf symbol placement in $[\mathrm{Fe} / \mathrm{H}]$ is representative and does not depict a preferred value (18). 